\title{
To induce or not to induce: the fight over hepcidin regulation
}

\author{
Veena Sangkhae and Elizabeta Nemeth \\ Center for Iron Disorders, David Geffen School of Medicine, University of California, Los Angeles, CA, USA \\ E-mail: NEMETH ELIZABETA - enemeth@mednet.ucla.edu
}

doi:10.3324/haematol.2019.216960

S ystemic iron homeostasis is co-ordinated by the hepatic hormone hepcidin. ${ }^{1}$ Hepcidin inhibits iron export through the cellular iron transporter ferroportin, thereby preventing iron absorption and the release of recycled or stored iron into plasma, resulting in decreased plasma iron levels. ${ }^{2}$ Hepcidin production changes rapidly and over a large dynamic range to ensure the maintenance of iron homeostasis. Hepcidin is suppressed in conditions that require increased iron supply, such as stress erythropoiesis, hypoxia, growth and pregnancy. ${ }^{3,4}$ Conversely, hepcidin is induced by iron loading to prevent the accumulation of excess iron, or by inflammation as part of the host defense response to infection. ${ }^{5,6}$ Although the regulation of hepcidin by singular stimuli has been well studied, particularly in animal models, we still do not have an understanding of the complex interplay of opposing signals in regulating hepcidin expression and iron homeostasis in humans.

In this issue of Haematologica, Stoffel et al. report on a prospective study in young women to evaluate the relative contribution of iron deficiency anemia and acute inflammatory stimulus on iron homeostasis ${ }^{7}$ (Figure 1). The well-controlled study included a total of 46 women: 25 non-anemic and 21 with iron-deficiency anemia (IDA). Compared to their non-anemic counterparts, anemic women had $2 \mathrm{~g} / \mathrm{dL}$ lower hemoglobin, lower serum iron, transferrin saturation, ferritin and body iron stores, and higher erythropoietin and serum transferrin receptor. The study excluded subjects with confounding factors affecting iron metabolism, including pre-existing inflammation, chronic disease, obesity, pregnancy, or vitamin and/or mineral supplementation for two weeks prior to and during the study. An acute inflammatory stimulus was modeled using an intramuscular injection of an influenza/diphtheria-tetanus-pertussis vaccine in all subjects. Inflammatory and iron markers were measured at baseline and 8, 24 and 36 hours (h) post vaccination.

The subjects also received test meals containing ${ }^{57} \mathrm{Fe}$ (nonradioactive isotope of iron), that allowed assessment of erythrocyte ${ }^{57} \mathrm{Fe}$ incorporation as a measure of iron absorption. The first ${ }^{57} \mathrm{Fe}$ meal and the first erythrocyte ${ }^{57} \mathrm{Fe}$ measurement were completed before the inflammatory stimulus ("baseline"). The second ${ }^{57} \mathrm{Fe}$ meal was administered $24 \mathrm{~h}$ after the vaccine, at the time of maximal or near-maximal IL6 and hepcidin increase, followed by the second erythrocyte ${ }^{57} \mathrm{Fe}$ measurement ["post-vaccine" (Figure 1)]. Although the erythrocyte ${ }^{57} \mathrm{Fe}$ measurements were performed 19 days after each ingestion of ${ }^{57} \mathrm{Fe}$, they should closely reflect iron absorption on the day of the meal consumption for the following reasons. In humans who are not iron-loaded, the majority of the absorbed iron is loaded onto transferrin and is destined for erythropoiesis: ferrokinetics experiments showed that following the ingestion of ${ }^{59} \mathrm{Fe}$, approximately $82-91 \%$ of absorbed radiolabeled iron is detected in erythrocytes after two weeks., Furthermore, erythrocyte lifespan is around 120 days, much longer than the duration of the
Stoffel et al. study. Thus, any confounding effect of ${ }^{57} \mathrm{Fe}$-red blood cell recycling and hepcidin modulation of the recycled iron flows would have been minimal.

Administration of the vaccine induced systemic inflammation in both cohorts of women, as reflected by an increase in interleukin-6 (IL-6), a major regulator of hepcidin production. Despite this, there was a surprising difference in the hepcidin response. Serum hepcidin increased in the non-anemic group within $24 \mathrm{~h}$ after vaccination but was unchanged in the IDA group. IL- 6 and hepcidin significantly correlated at $24 \mathrm{~h}$ after vaccination only in the non-anemic but not in the IDA group. Serum iron levels mirrored the hepcidin response: in the non-anemic cohort, increased serum hepcidin was associated with decreased serum iron, whereas in the IDA group, no change in serum iron was observed. The authors therefore concluded that during IDA, regulation of hepcidin by iron and/or erythropoietic activity supersedes hepcidin regulation by acute inflammation. Measurement of erythrocyte iron incorporation from ${ }^{57} \mathrm{Fe}$-labeled test meals provided a valuable insight into iron absorption before and after the acute inflammatory stimulus. Erythrocyte iron incorporation was higher in IDA compared to non-anemic subjects at all time points examined, reflecting increased iron absorption in this group. Interestingly, erythrocyte ${ }^{57} \mathrm{Fe}$ incorporation was not affected by inflammation in either group, despite increased hepcidin in the non-anemic women. As the authors point out, a possible explanation is that enterocytes may be less sensitive to the effect of hepcidin than recycling macrophages. ${ }^{10}$ Thus, a moderate increase in hepcidin after vaccination in non-anemic women could cause hypoferremia without, at the same time, affecting duodenal ${ }^{57} \mathrm{Fe}$ absorption, because serum iron concentration is predominantly determined by macrophage iron export. Interestingly, in the non-anemic group, erythrocyte ${ }^{57} \mathrm{Fe}$ incorporation was inversely correlated with serum hepcidin both at baseline $(\mathrm{r}=-0.792 ; P<0.001)$ and after vaccination $(\mathrm{r}=-0.708 ; P<0.001)$. This suggests that, over a broader range of concentrations, hepcidin does modulate iron absorption, but that hepcidin changes after the vaccination were too small to exert an effect on enterocytes.

This study is the first to test the dynamic hierarchical regulation of hepcidin by iron and inflammation in a well-controlled trial in humans, and showed that iron-deficiency anemia exerted a dominant effect over that of acute inflammation in this setting. What is the molecular mechanism that could explain this observation? The hepcidin promoter contains both bone morphogenetic protein (BMP)-response elements (RE) and a STAT3-RE. ${ }^{11}$ Iron-mediated hepcidin regulation occurs via the BMP-SMAD pathway. It is thought that liver sinusoidal endothelial cells secrete BMP2 and BMP6 in proportion to the liver iron stores; ${ }^{12,13}$ these ligands then act in a paracrine fashion, and bind $\mathrm{BMP}$ receptors and their coreceptor hemojuvelin (HJV) on hepatocytes to induce phosphorylation of SMAD1/5. Phosphorylated SMAD1/5 form a 
A
Intervention
test-meal
$57 \mathrm{Fe}$
$\underset{57 \mathrm{Fe}}{\operatorname{vaccine}}$ test-meal
13
$57 \mathrm{Fe}$

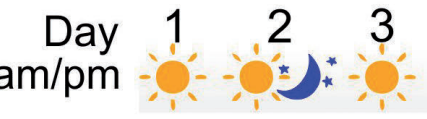
$22 \quad 23 \quad 24$
45

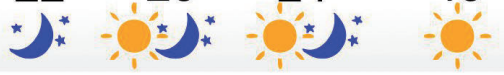
Biochemistry
$\mathrm{pm}$ am $8 \mathrm{~h} 24 \mathrm{~h} 36 \mathrm{~h}$
baselines post-vaccine
$57 \mathrm{Fe}$
baseline

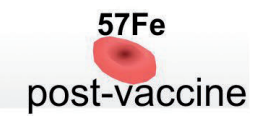

Erythrocyte ${ }^{57} \mathrm{Fe}$
incorporation

B

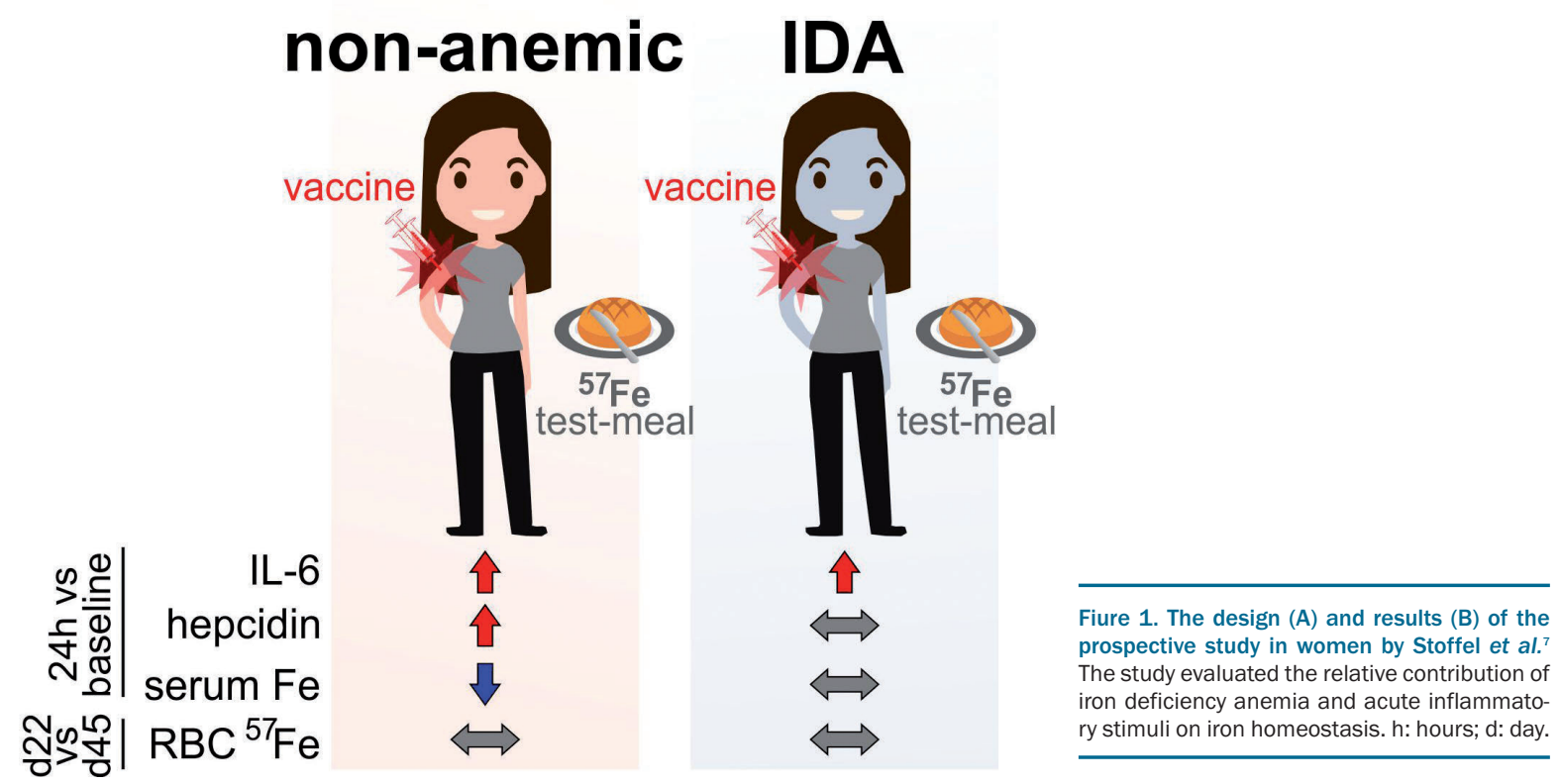

complex with SMAD4, translocate to the hepatocyte nucleus, and bind to the BMP-RE to induce hepcidin expression. Holo-transferrin concentrations, which are sensed by the TfR1/HFE and TfR2 proteins on hepatocytes, are also thought to modulate the same BMP signaling pathway in these cells. Low iron stores and low circulating iron (observed in the IDA group in this study), would result in decreased BMP signaling and a low level of hepcidin transcription. Low hepcidin would then allow for increased iron absorption and mobilization from stores. However, in the presence of infection, increased iron bioavailability becomes a liability, as pathogens also require iron for proliferation and survival. As part of the host defense, hepcidin is induced by infection and inflammation to limit iron availability to pathogens. Hepcidin regulation by infection and inflammation is mediated in large part by IL- $6 .{ }^{14} \mathrm{IL}-6$ binding to its receptor, IL-6R $\alpha$, and co-receptor, gp130, results in phosphorylation of JAK $1 / 2$ in hepatocytes, which then phosphorylates STAT3. This then dimerizes and translocates to the nucleus to induce hepcidin expression.

Importantly, the BMP pathway was shown to synergize with STAT3 pathway to induce hepcidin transcription.
Disruption of BMP-RE in a liver cell line impaired hepcidin response to IL- $6 .{ }^{15}$ Studies using mouse models have demonstrated that hepcidin induction in response to inflammation is blunted when hepatic BMP signaling is genetically disrupted. ${ }^{16-20}$ Absence of HJV or ALK3 prevented induction of hepcidin in vivo following acute inflammatory stimulus (LPS or IL-6). ${ }^{17}$ Similarly, in Hfe and Tfr 2 knockout mice, hepcidin induction in response to LPS was also blunted. ${ }^{16}$ Although these mouse studies did not model iron-deficiency anemia, such as was seen in the study subjects in Stoffel et al. ${ }^{7}$ they provided the proof of principle that the BMP pathway plays an important role in hepcidin responsiveness to inflammation.

However, it remains to be determined whether the presumed decrease in BMP-SMAD signaling in the study subjects is caused by iron deficiency, or by anemia and increased erythropoietic activity, or the combination of these factors. Anemia induces erythropoietin (EPO) secretion by the kidneys. ${ }^{21} \mathrm{EPO}$ in turn acts on the bone marrow erythroblasts to induce expression of erythroferrone (ERFE), ${ }^{22}$ and ERFE is shown to function as a BMP trap to suppress hepcidin. ${ }^{23}$ Although EPO was elevated in IDA subjects in this study, serum ERFE levels were not meas- 
ured but could provide insight into the contribution of anemia to the blunted hepcidin response. It would be interesting to see if iron deficiency alone is sufficient to prevent hepcidin induction following acute inflammatory stimulation. In fact, eight of the 25 women in the nonanemic group were reported to be iron-deficient, but were not analyzed as a subgroup to determine the contribution of anemia versus iron deficiency.

In addition to the convergence of signals onto the hepcidin promoter, another aspect to consider in the regulation of hepcidin is the relative strength and duration of each signal. In this study, iron-deficiency anemia was relatively mild (median hemoglobin of $11.3 \mathrm{~g} / \mathrm{dL}$ ), but likely chronic. The inflammatory signal was moderate and likely transient, with IL-6 increasing approximately 2-3-fold after vaccination compared to baseline. Hepcidin induction was similarly moderate: in the non-anemic group, hepcidin levels increased 2 -fold by $24 \mathrm{~h}$ compared to baseline. Whether a stronger or more prolonged inflammatory stimulus, such as during an active infection, would over-ride the effect of IDA on hepcidin remains to be determined. Nevertheless, in agreement with Stoffel et al., a cross-sectional study that compared patients with anemia of chronic disease (ACD) to those with IDA or mixed ACD/IDA condition, reported that hepcidin was increased in patients with ACD compared to control subjects, but that in patients with mixed ACD/IDA, despite elevated IL-6, hepcidin levels were comparable to those observed in IDA patients. ${ }^{24}$

In conclusion, the data obtained from the welldesigned and well-executed prospective study in human subjects by Stoffel et al. support the conclusion that during iron-deficiency anemia, when challenged by moderate but transient acute inflammation, iron acquisition is prioritized over iron restriction. The questions about the molecular mechanism and relative contribution of erythropoietic activity versus iron deficiency in preventing an inflammation-mediated increase in hepcidin still have to be answered. Importantly, this human study pioneers the analysis of the interactions of iron deficiency and inflammation, a subject of great importance for designing and implementing policies to prevent and treat anemia in regions where iron deficiency, infection and inflammation are all too common.

\section{Acknowledgments and disclosures}

Sources of support: NIH Ruth L. Kirschstein National Research Service Award T32-5T32HL072752-13 (to VS). EN is a shareholder and scientific advisor of Intrinsic LifeSciences.

\section{References}

1. Ganz T. Systemic iron homeostasis. Physiol Rev. 2013;93(4):17211741.

2. Nemeth E, Tuttle MS, Powelson J, et al. Hepcidin regulates cellular iron efflux by binding to ferroportin and inducing its internalization.
Science. 2004;306(5704):2090-2093.

3. Sangkhae V, Nemeth E. Regulation of the Iron Homeostatic Hormone Hepcidin. Adv Nutr. 2017;8(1):126-136.

4. Nicolas G, Chauvet C, Viatte L, et al. The gene encoding the iron regulatory peptide hepcidin is regulated by anemia, hypoxia, and inflammation. J Clin Invest. 2002;110(7):1037-1044.

5. Cassat JE, Skaar EP. Iron in infection and immunity. Cell Host Microbe. 2013;13(5):509-519.

6. Arezes J, Jung G, Gabayan V, et al. Hepcidin-induced hypoferremia is a critical host defense mechanism against the siderophilic bacterium Vibrio vulnificus. Cell Host Microbe. 2015;17(1):47-57.

7. Stoffel NU, Lazrak M, Bellitir S, et al. The opposing effects of acute inflammation and iron deficiency anemia on serum hepcidin and iron absorption in young women. Haematologica. 2019;104(6):11431149

8. Marx JJ, Dinant HJ. Ferrokinetics and red cell iron uptake in old age: evidence for increased liver iron retention? Haematologica. 1982;67(2):161-168.

9. Marx JJ. Normal iron absorption and decreased red cell iron uptake in the aged. Blood. 1979;53(2):204-211.

10. Chaston T, Chung B, Mascarenhas M, et al. Evidence for differentia effects of hepcidin in macrophages and intestinal epithelial cells Gut. 2008:57(3):374-382

11. Truksa J, Lee P, Beutler E. Two BMP responsive elements, STAT, and bZIP/HNF4/COUP motifs of the hepcidin promoter are critical for BMP, SMAD1, and HJV responsiveness. Blood. 2009;113(3):688-695.

12. Canali S, Zumbrennen-Bullough $\mathrm{KB}$, Core AB, et al. Endothelial cells produce bone morphogenetic protein 6 required for iron homeostasis in mice. Blood. 2017;129(4):405-414

13. Koch PS, Olsavszky V, Ulbrich F, et al. Angiocrine Bmp2 signaling in murine liver controls normal iron homeostasis. Blood. 2017;129(4):415-419.

14. Nemeth E, Rivera S, Gabayan V, et al. IL-6 mediates hypoferremia of inflammation by inducing the synthesis of the iron regulatory hormone hepcidin. J Clin Invest. 2004;113(9):1271-1276.

15. Verga Falzacappa MV, Casanovas G, Hentze MW, Muckenthaler MU. A bone morphogenetic protein (BMP)-responsive element in the hepcidin promoter controls HFE2-mediated hepatic hepcidin expression and its response to IL- 6 in cultured cells. J Mol Med. 2008;86(5):531-540.

16. Wallace DF, McDonald CJ, Ostini L, Subramaniam VN. Blunted hepcidin response to inflammation in the absence of Hfe and transferrin receptor 2. Blood. 2011;117(10):2960-2966

17. Fillebeen C, Wilkinson N, Charlebois E, Katsarou A, Wagner J, Pantopoulos K. Hepcidin-mediated hypoferremic response to acute inflammation requires a threshold of $\mathrm{Bmp} 6 / \mathrm{Hjv} / \mathrm{Smad}$ signaling. Blood. 2018;132(17):1829-1841.

18. Huang H, Constante M, Layoun A, Santos MM. Contribution of STAT3 and SMAD4 pathways to the regulation of hepcidin by opposing stimuli. Blood. 2009;113(15):3593-3599.

19. Steinbicker AU, Sachidanandan C, Vonner AJ, et al. Inhibition of bone morphogenetic protein signaling attenuates anemia associated with inflammation. Blood. 2011;117(18):4915-4923.

20. Mayeur C, Lohmeyer LK, Leyton P, et al. The type I BMP receptor Alk3 is required for the induction of hepatic hepcidin gene expression by interleukin-6. Blood. 2014;123(14):2261-2268.

21. Haase VH. Regulation of erythropoiesis by hypoxia-inducible factors. Blood Rev. 2013;27(1):41-53.

22. Kautz L, Jung G, Valore EV, Rivella S, Nemeth E, Ganz T. Identification of erythroferrone as an erythroid regulator of iron metabolism. Nature Genet. 2014;46(7):678-684.

23. Arezes J, Foy N, McHugh K, et al. Erythroferrone inhibits the induction of hepcidin by BMP6. Blood. 2018;132(14):1473-1477.

24. Theurl I, Aigner E, Theurl M, et al. Regulation of iron homeostasis in anemia of chronic disease and iron deficiency anemia: diagnostic and therapeutic implications. Blood. 2009;113(21):5277-5286. 